\title{
BMJ Open Surgeon perceived most important factors to achieve the best hospital performance on colorectal cancer surgery: a Dutch modified Delphi method
}

Julia Tessa van Groningen, ${ }^{\oplus 1,2}$ Perla J Marang-van de Mheen, ${ }^{3}$ Daniel Henneman, ${ }^{1}$ Geerard L Beets, ${ }^{4}$ Michel W J M Wouters ${ }^{2,4}$

To cite: van Groningen JT, Marang-van de Mheen PJ, Henneman D, et al. Surgeon perceived most important factors to achieve the best hospital performance on colorectal cancer surgery: a Dutch modified Delphi method. BMJ Open 2019;9:e025304. doi:10.1136/ bmjopen-2018-025304

- Prepublication history for this paper is available online. To view these files, please visit the journal online (http://dx.doi org/10.1136/bmjopen-2018025304).

Received 9 July 2018

Revised 18 June 2019 Accepted 1 July 2019
Check for updates

(C) Author(s) (or their employer(s)) 2019. Re-use permitted under CC BY-NC. No commercial re-use. See rights and permissions. Published by BMJ.

For numbered affiliations see end of article.

Correspondence to

Dr Julia Tessa van Groningen; julia.vangroningen@gmail.com

\section{ABSTRACT}

Objectives Hospital variation in risk-adjusted outcomes after colorectal cancer surgery has been shown. However, explanatory factors are not sufficiently clear. The objective of this study was to identify factors perceived by gastrointestinal surgeons as important to achieve excellent casemix-adjusted outcomes after colorectal cancer surgery.

Design Based on literature and experts' opinion, 86 factors associated with serious complications, failure to rescue and mortality were listed. These were presented to gastrointestinal surgeons through two web-based surveys and an expert meeting. Participants were asked to choose their top 10 of most important factors.

Participants Dutch gastrointestinal surgeons $(n=52)$ of different hospitals and different hospital types (general/ teaching/academic).

Results of 31 invited experts for the first survey and meeting, $71 \%$ responded. Of 130 invited surgeons, 34 responded to the second survey. Factors deemed important were: procedural hospital volume (46\% in top 10 ), specialised surgeons performing surgery, (elective $87 \%$, emergency $60 \%$ and reoperations $62 \%$ in top 10 ), accessibility of, and daily ward rounds by specialised surgeons ( $41 \%$ and $38 \%$ in top 10$)$, preoperative screening for malnutrition ( $57 \%$ in top 10$)$, a protocol for recognition of anastomotic leakage and rapid reintervention ( $54 \%$ and $49 \%$ in top 10).

Conclusion Procedural hospital volume, specialisation of surgeons, screening for malnutrition, early recognition of complications followed by rapid action were perceived as most important factors to achieve good outcomes by gastrointestinal surgeons.

\section{INTRODUCTION}

Hospital variation in surgical outcomes is receiving increasing attention. Nowadays, in many countries, quality of surgical care is assured by concentration of care, minimum care standards, increasing specialisation of surgeons and clinical audit projects. Still, variation in casemix-adjusted outcomes between
Strengths and limitations of this study

- This study was designed find consensus among gastrointestinal surgeons about important factors leading to the best performance in colorectal cancer surgery, an important first step in targeting quality improvement initiatives.

- Strengths of this study are the extensive questioning, in different rounds and in different manners, increasing the reliability of the identified most important factors.

- Extensive questioning also creates a risk of low response rates.

hospitals remains and mechanisms behind this are not completely understood. ${ }^{1-3}$

Traditionally, in high-complex and low-volume procedures, variation in postoperative mortality was approached by centralisation of care; reducing complications by increasing hospital procedural volumes. This volume-outcome relationship proved to be less strong in more commonly performed colorectal cancer surgery. ${ }^{45}$ Literature shows associations between outcomes and some structural factors, for example, level of intensive care unit, nurse staffing or hospital procedural volume. However, the best-performing hospitals differ in their structural characteristics, and structural factors alone do not explain hospital variation in the outcomes. ${ }^{6-9}$

Simultaneous to centralisation efforts, many countries initiated the clinical audits. Their aim was stimulating quality improvement projects in individual hospitals by benchmarking outcomes. In the Netherlands, the Dutch Surgical Colorectal Audit (DSCA) measures and feeds back processes and casemix-adjusted outcomes to all hospitals performing colorectal cancer surgery. ${ }^{10}$ 
Furthermore, an expert team visits hospitals with significantly more serious complications or mortality than average, aiming to initiate an improvement cycle. Over the last years, the Dutch serious complication, 'failure to rescue' (FTR) and mortality rates improved, although variation between hospitals remains. ${ }^{23}$

In addition to structural factors, many procedural factors could explain the variation in outcomes, such as differences in postoperative monitoring and time to recognition. ${ }^{1}{ }^{11-13}$ Furthermore, different healthcare professionals are involved in perioperative patient care: nurses, paramedical personnel and different medical specialists. Hence, teamwork, culture and communication between these professionals may be important. ${ }^{11}$ Another important factor could be the increasing role of specialised gastrointestinal surgeons rather than the general surgeon, in surgery, but also in postoperative management and daily ward rounds. ${ }^{14}$

Which factors are the most important in relation to the observed variation in outcomes is not sufficiently clear. In unravelling this process of care in good performing hospitals, the first step is identifying factors related to differences in outcomes in a structured fashion. ${ }^{15-17}$ This study aims to identify factors that could be related to better or worse outcomes in colorectal cancer surgery and to identify those factors that gastrointestinal surgeons perceive as most important to explain hospital differences in outcomes.

\section{METHODS}

A modified Delphi method was conducted to collect opinions of Dutch surgeons participating in the DSCA (figure 1). The outcomes of interest were: serious complications, FTR and postoperative mortality. Serious complications were defined as complications leading to, prolonged in-hospital stay ( $>14$ days), a reintervention or mortality. FTR was defined as the percentage of patients dying after a serious complication. ${ }^{18}$ Two web-based surveys $^{19}$ and one expert meeting were held between August 2014 and June 2015. After a literature search for potential influencing factors, a comprehensive list comprised. The first web-based survey (round 1) and the expert meeting (round 2) were used to shorten the initial list of factors. The second web-based survey (round 3) was used to test perceived importance of selected factors among a larger group of gastrointestinal surgeons. The respondents were invited by email, one reminder sent in case of non-response. Both web-based surveys were open for 3 months. Informed consent from respondents was given at the start of the survey.

\section{Literature search}

First, literature was searched for factors related to shortterm outcomes after colorectal cancer surgery. The search was done in PubMed and Embase. Search terms used were: (morbidity OR mortality OR postoperative complications OR Failure to rescue) AND (Colorectal

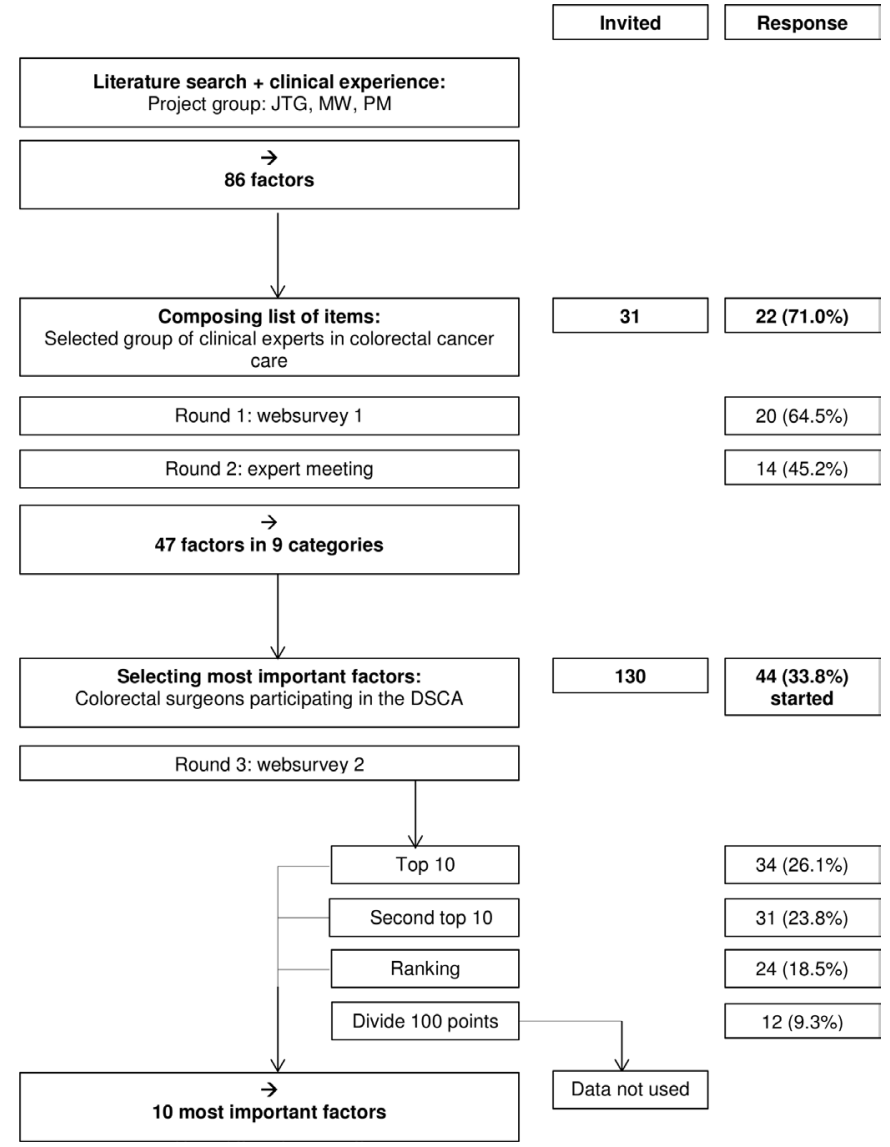

Figure 1 Modified Delphi method. DSCA, Dutch Surgical Colorectal Audit.

Surgery OR ((surgery OR surgical OR operative OR operation) AND (colon OR colonic OR rectal OR rectum OR colorectal)). Additional literature searches were done for factors derived from clinical experience. After this initial step, the list contained 86 factors, which were grouped into nine categories.

\section{Round 1: web-based survey 1}

The 86 factors were presented to a selected group $(n=31)$ of (mandated) clinical experts in colorectal cancer care through a web-based survey. The respondents were first asked to select their top 10 of most important factors, and to divide 100 points between the factors mentioned in the top 10. Second, they were asked to rank the factors within nine categories on the extent to which factors would vary between hospitals and would be relevant for the outcomes: serious complications, FTR and postoperative mortality. The nine categories covered preoperative, intraoperative and postoperative processes, the process of diagnosis and treatment of complications, structural hospital factors and factors involving the healthcare providers, including their accessibility outside working hours, communication and level of education. One was defined as the highest rank; the lowest rank was dependent on the number of factors present in each category (varying from 8 to 10). Last, the respondents were asked to select their top 10 
again, as seeing all factors in detail might have changed their assessment.

\section{Round 2: expert meeting}

Results of the first web-based survey were presented during the expert meeting. Of the 31 invited experts, 14 were present at this meeting. Every category was presented and factors were ordered based on a combination of the perceived extent of variation between hospitals and perceived importance for outcomes. Mean rankings within a category for each factor were shown, if this was higher than the median in that category, or if a factor was mentioned in a top 10 , it was considered relevant for the next round. The experts had the possibility to switch factors from relevant to irrelevant or vice versa and the possibility to change the categories to which the factors were assigned. If new factors were mentioned during the meeting, consensus was found to decide if these were added to a suitable category.

After the expert meeting, the list consisted of 47 factors divided into 9 (new) categories: preoperative, intraoperative (elective and emergency setting) and postoperative processes, communication, the team of healthcare providers and their accessibility during, and outside working hours, and structural hospital factors.

\section{Round 3: web-based survey 2}

The second web-based survey was distributed to all surgeons $(n=130)$ participating in the DSCA. This survey questioned the importance of the 47 factors explaining the hospital variation in outcomes. Respondents were specifically asked to select factors explaining variation between hospitals, so they would not choose factors that are embedded in all Dutch hospitals (eg, enhanced recovery protocols). Again, respondents were asked to select a top 10. Additionally, respondents had the option to divide 100 points among the factors in their top 10 and to rank the factors within their 9 categories.

\section{Analyses}

The percentage of respondents selecting a factor in their top 10 was calculated. The factors were ordered based on this percentage from high to low and we selected the ten factors with the highest percentage in the top 10 as most important factors. Potential differences assigned by surgeons working in general hospitals and in academic (affiliated) hospitals were tested using the $\mathrm{X}^{2}$ test.
The additional questions were used to calculate a sum of points assigned to a factor and the mean rank (MR) within its category and to order the factors again based on these outcomes. Statistical analyses were performed in IBM SPSS Statistic V.22.

\section{Patient and public involvement}

Patients were not involved in this study.

\section{RESULTS}

A total of $52(40 \%)$ gastrointestinal surgeons participated in one or more rounds of the modified Delphi method (table 1). Of these respondents, 13 were working in academic, 31 in teaching and 11 in general hospitals. In the first two rounds, the response rate was $71 \%$ and in the last round, the response rate was $26 \%$ (table 1 ).

\section{Round 1 and 2: composing a list of factors}

All new mentioned factors in the first web-based survey were included in our analyses. From the 86 factors initially selected by the project group and 15 new factors, 47 were selected for the next round based on consensus among the experts. Some factors were deleted and some were adapted or merged with another factor. The resulting list of 47 factors across 9 categories was used in the second web-based survey and is presented in table 2 .

\section{Round 3: identifying most important factors}

Forty-four surgeons started the second web-based survey, 34 surgeons selected their top 10 (figure 1 ). Table 2 shows the percentage of surgeons that selected the factor in the top 10, sorted from highest to lowest importance within their category (10 most important factors marked with *). Our consensus shows 1-3 important factors in seven of the nine categories. In the categories 'communication' and 'healthcare providers', no factor was selected.

Only one factor differed significantly between surgeons working in general hospitals and surgeons working in academic (affiliated) hospitals. The accessibility of a specialised surgeon to also examine a patient during business hours was mentioned in the top 10 by more surgeons in general hospitals (67\%) compared with surgeons in academic (affiliated) hospitals (28\%, $\mathrm{p}=0.025)$. Other factors did not differ significantly, indicating broad consensus on most important factors regardless of work setting.

\begin{tabular}{llllll}
\hline Table 1 Number of respondents in all rounds & & & & \\
& Invited & Total response & Academic & Teaching & General \\
\hline Composing list of items total & 31 & $22(71 \%)$ & 9 & 13 & 0 \\
$\quad$ Websurvey 1 only (round 1) & & 8 & & & \\
$\quad$ Expert meeting only (round 2) & & 2 & & & \\
$\quad$ Both round 1 and 2 & 12 & $34(26 \%)$ & 5 & 18 & 11 \\
Selecting most important items total & 130 & $52(40 \%)$ & 13 & 28 & 11 \\
Total participants & 130 & & \\
\hline
\end{tabular}


Table 2 Percentage of respondents reporting a factor in their top 10, sum of points assigned and mean rank within category

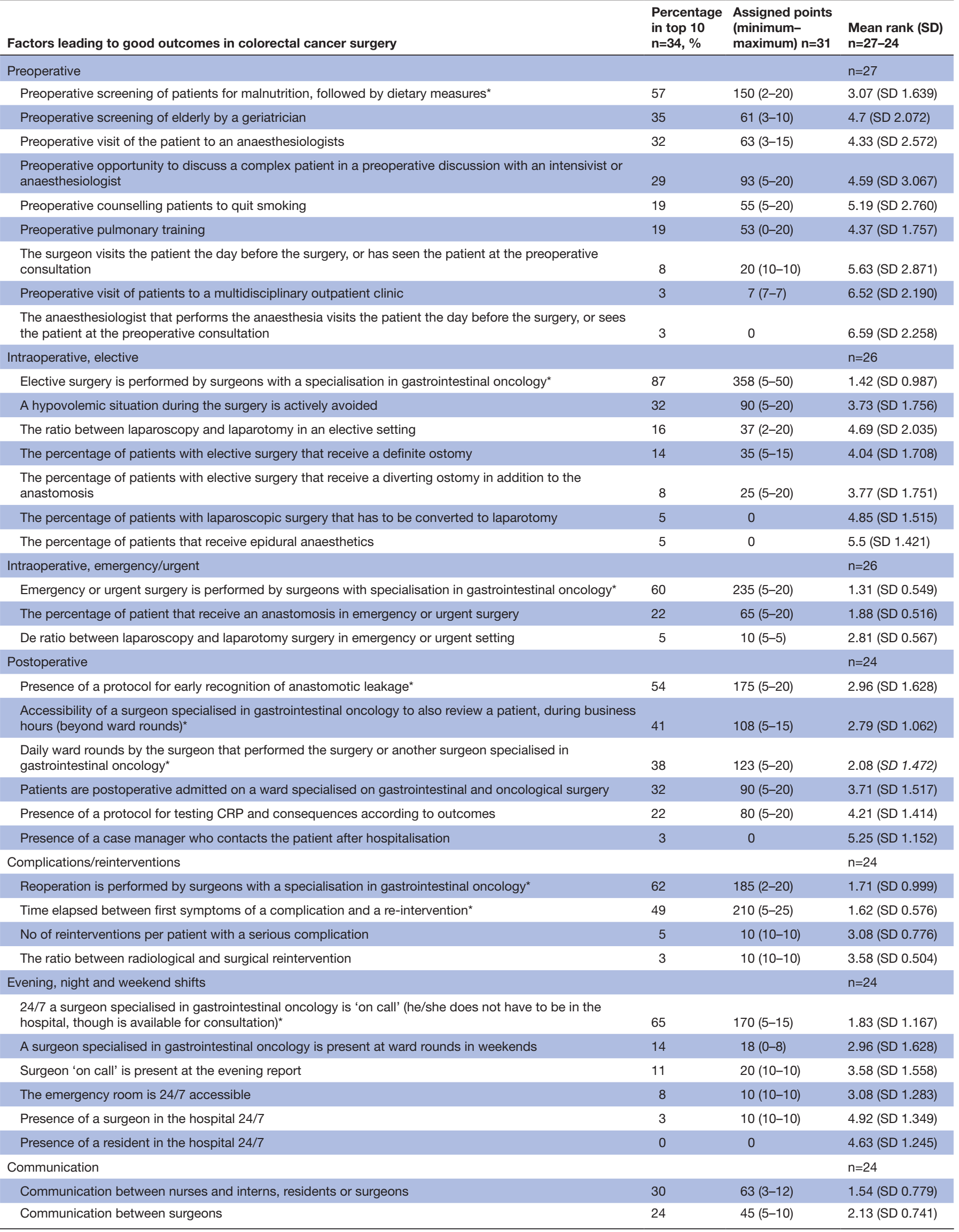


Table 2 Continued

Factors leading to good outcomes in colorectal cancer surgery

\begin{tabular}{|c|c|c|c|}
\hline Factors leading to good outcomes in colorectal cancer surgery & $\begin{array}{l}\text { Percentage } \\
\text { in top } 10 \\
n=34, \%\end{array}$ & $\begin{array}{l}\text { Assigned points } \\
\text { (minimum- } \\
\text { maximum) } n=31\end{array}$ & $\begin{array}{l}\text { Mean rank (SD) } \\
n=27-24\end{array}$ \\
\hline At least monthly discussion of outcomes (including discussion of complications) & 19 & $55(5-15)$ & 3.46 (SD 0.932) \\
\hline Communication between surgeon and anaesthesiologist & 8 & $15(5-10)$ & 2.88 (SD 0.992) \\
\hline Healthcare providers & & & $n=24$ \\
\hline Average experience of nurses on the wards & 30 & $70(2-20)$ & 1.75 (SD 0.737) \\
\hline Average experience of interns and residents responsible for the wards & 11 & $18(8-10)$ & 2.63 (SD 1.173) \\
\hline The hospital is a referral centre for colorectal surgery & 5 & $5(5-5)$ & 2.75 (SD 1.073) \\
\hline No of nurses per patient (nurse/patient ratio) & 5 & $5(5-5)$ & 2.88 (SD 1.154) \\
\hline No of surgeons specialised in gastrointestinal oncology in a hospital & 5 & $10(10-10)$ & 4.29 (SD 1.628) \\
\hline Hospital structure & & & $\mathrm{n}=24$ \\
\hline No of colorectal surgeries (both benign and malignant) performed in the hospital annually* & 46 & $103(2-20)$ & 2.42 (SD 1.586) \\
\hline Presence of emergency intervention team & 24 & $53(2-15)$ & 2.54 (SD 1.668) \\
\hline Accessibility of an intervention radiologist & 19 & $35(5-10)$ & 4.17 (SD 1.633) \\
\hline The ICU level of the hospital & 11 & $30(5-20)$ & 4.08 (SD 1.381) \\
\hline The operating team also performs other high-complex surgeries & 8 & $17(2-10)$ & $3.5(\mathrm{SD} 1.445)$ \\
\hline
\end{tabular}

*Ten most important factors are marked with.

$\mathrm{CRP}, \mathrm{C}$ reactive protein; ICU, intensive care unit.

Additionally, 31 surgeons assigned points to the factors in their top 10 (total 3100 points) and, 24 surgeons ranked the factors in all categories. The sum of assigned points and MRs (including SD) are shown in table 2. The main analyses and these subanalyses resulted in the same 10 most important factors (marked with * in table 2 and shown in box 1).

Figure 2 shows the strong association between percentage of respondents selecting a factor in their top 10 and MR assigned to this factor. It shows all most

Box 1 Surgeons perceived 10 most important factors to achieve the best hospital performance on colorectal cancer surgery

Elective surgery is performed by surgeons with a specialisation in gastrointestinal oncology.

- 24/7 a surgeon specialised in gastrointestinal oncology is 'on call' (he/she does not have to be in the hospital, though is available for consultation).

- Reoperation is performed by surgeons with a specialisation in gastrointestinal oncology.

- Emergency or urgent surgery is performed by surgeons with specialisation in gastrointestinal oncology.

- Preoperative screening of patients for malnutrition followed by dietary measures.

- Presence of a protocol for early recognition of anastomotic leakage.

- Time elapsed between first symptoms of a complication and a reintervention.

- Number of colorectal surgeries (both benign and malignant) performed in the hospital annually.

- Accessibility of a surgeon specialised in gastrointestinal oncology to also review a patient, during business hours (beyond ward rounds).

- Daily ward rounds by the surgeon that performed the surgery or another surgeon specialised in gastrointestinal oncology. important factors in the top right part of the figure, indicating a combination of high ranks (highest=1) and high percentages mentioning the factor in the top 10 .

\section{DISCUSSION}

This modified Delphi method identified 10 factors deemed as most important by Dutch gastrointestinal surgeons in explaining variation in casemix-adjusted outcomes after colorectal cancer surgery. Procedural hospital volume was selected as an important structural factor. In six of the most important factors, the specialised surgeon has an important role. In the preoperative phase, screening for malnutrition was considered important; in the postoperative phase, fast recognition of a complication followed by a rapid reintervention and a protocol for early recognition of anastomotic leakage. In total, 10 factors were selected as most important.

Comparing our results to the literature, it is striking that surgeons perceive procedural hospital volume as one of the most important factors. While procedural hospital volume has often been considered a proxy for high quality of care in high-complex and low-volume procedures, the evidence in colorectal surgery is less convincing. Several systematic reviews show a volume-outcome relationship in colorectal cancer surgery. However, the effect sizes are small, it is a non-linear effect and the definition of high hospital volume is heterogenic between different studies. This makes it hard to define a clear threshold for minimum annual number of colorectal cancer surgeries per hospital. ${ }^{20-24}$ Also, a difference between US and non-US data suggests that hospital level variability was less of a problem outside the USA than in the USA. ${ }^{2024}$ Furthermore, Dutch studies show that volume is no guarantee for 


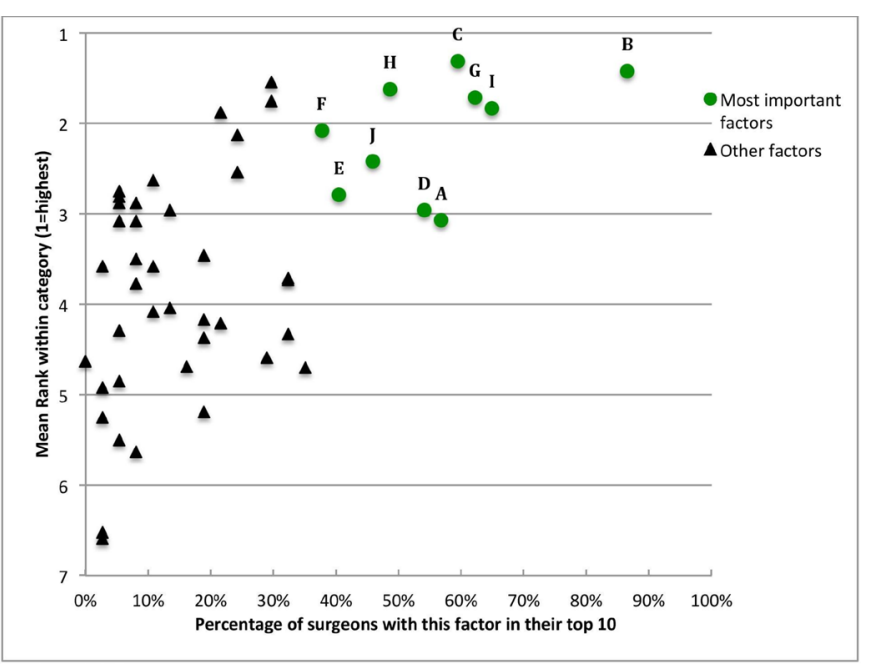

Figure 2 MR within category by percentage of surgeons selecting this factor in their top 10. Most important factors are: (A) Preoperative screening of patients for malnutrition, followed by dietary measures; (B) Elective surgery is performed by surgeons with a specialisation in gastrointestinal oncology; (C) Emergency or urgent surgery is performed by surgeons with specialisation in gastrointestinal oncology; (D) Presence of a protocol for early recognition of anastomotic leakage; (E) Accessibility of a surgeon specialised in gastrointestinal oncology to also review a patient, during business hours (beyond ward rounds); (F) Daily ward rounds by the surgeon that performed the surgery or another surgeon specialised in gastrointestinal oncology; (G) Reoperation is performed by surgeons with a specialisation in gastrointestinal oncology; (H) Time elapsed between first symptoms of a complication and a reintervention; (I) 27/7 a surgeon specialised in gastrointestinal oncology is 'on call' (he/she does not have to be in the hospital, though is available for consultation) (J) Number of colorectal surgeries (both benign and malignant) performed in the hospital annually. MR, mean rank.

optimal outcomes in colorectal cancer care in the Netherlands. $^{25} 26$ More importantly, investigating structural hospital factors only will not reveal differences in patterns of care that lead to good outcomes. ${ }^{25} 26$

In addition to procedural hospital volume, the effect of individual surgeon volume and specialisation of the surgeons seems more important. ${ }^{20} 21$ Respondents in our study strongly expressed that specialisation of the surgeons performing colorectal cancer resections is associated with better outcomes. This was in accordance with studies reporting better survival, but also a better adherence to guidelines and a better use of appropriate surgical techniques. ${ }^{27} 28$ The postoperative presence and accessibility of specialised surgeons and daily presence at ward rounds is considered important by our respondents, although there is little evidence substantiating this. ${ }^{14}$ The surgeons perceive that specialisation helps in installing preventive measures, and early recognition and better treatment of complications. Literature shows that for fast recognition and fast treatment, teamwork, communication and culture is important, and that the role of nurses and doctors of the ward is pivotal. ${ }^{11} 2930$ Interestingly, in our study respondents did not choose the factors on 'communication' or 'experience of healthcare providers' as most important. However, respondents found adherence to postoperative protocols important, which could be another way to regulate preventive measures, early recognition and better treatment of complications. These postoperative protocols for recognition of anastomotic leakage as well as standardised care programmes are developed to improve patient safety and surgical outcomes. $^{3132}$ Adherence to these protocols is not well known and can very well differ between hospitals. Better adoption of programmes and protocols that have shown to improve postoperative outcomes could lead to an improvement of colorectal cancer care. ${ }^{33} 34$

With an increasing population of elderly patients and a high prevalence of malnutrition in colorectal cancer patients, risk assessment and personalised preparation is increasingly important. Risk assessment is mostly done with the American Society of Anesthesiologist classification, but also specific scores for screening on malnutrition are used, for example, malnutrition universal screening tool or Short Nutritional Assessment Questionnaire. Our respondents perceive screening on malnutrition as highly important. Future data collection on these factors should show the extent to which this explains the observed variation in outcomes between hospitals.

There are limitations to this study. First, $40 \%$ of the invited surgeons participated in one or more rounds of this study, but $26 \%$ participated in the last round. Although these percentages are common in surveys among professionals, this could imply selection bias. ${ }^{35}$ However, the respondents were distributed across different types of hospitals (general and academic (affiliated) hospitals) in the same way this is distributed in the non-responders. This makes it plausible that our responders could reflect the beliefs of their (non-responding) colleagues. Second, we have no information on the experience of the surgeons. Still, because the invited surgeons were participating in the DSCA, interest is expected, and specialisation in colorectal cancer surgery is likely. In this study, surgeons were invited and no nurses or doctors of the wards, which might be reflected in the answers given. Perspectives of other healthcare providers and even patients could result in selection of other factors than the ones listed in this study. However, because surgeons make most perioperative decisions and are up to date on available evidence regarding colorectal surgery, they were thought to have the best expertise to judge which factors might explain the observed variation in outcomes between hospitals. Certainly, this study did not address all factors possibly influencing outcomes in colorectal cancer care (eg, discharge follow-up or innovative technologies being offered). However, the surgeons had the possibility to add new factors, which they thought to be important, in the expert meeting and those factors were included in the study. Furthermore, we investigated the opinion of Dutch gastrointestinal surgeons. Still, we feel that this study could be applicable to other countries with 
comparable processes already implemented nationwide. Last, we decided to select 10 most important factors. This cut-off point is arbitrary.

\section{CONCLUSION}

According to Dutch surgeons, important factors to achieve low morbidity and mortality, and to explain hospital variation in casemix-adjusted short-term postoperative outcomes after colorectal cancer surgery are: procedural hospital volume, specialisation of surgeons, screening for malnutrition and early recognition of complications followed by rapid action. More research is needed to determine to what extent the hospital variation in outcomes of colorectal cancer surgery in the Netherlands is explained by these factors. The consensus present on these factors could form the basis for further in-depth research on possible differences between hospitals with better and worse outcomes.

\section{Author affiliations}

${ }^{1}$ Department of Surgery, Leids Universitair Medisch Centrum, Leiden, The Netherlands

${ }^{2}$ Dutch Institute for Clinical Auditing, Leiden, The Netherlands

${ }^{3}$ Department of Biomedical Data Sciences, Medical Decision Making, Leids Universitair Medisch Centrum, Leiden, The Netherlands

${ }^{4}$ Department of Surgical Oncology, Antoni van Leeuwenhoek Nederlands Kanker Instituut, Amsterdam, The Netherlands

Acknowledgements The authors thank all the surgeons participating in the modified Delphi method.

Contributors Study concepts: MW, DH and PM. Study design: MW, DH and PM. Data acquisition: JTG, MW, DH, PM and GB. Quality control of data and algorithms: JTG. Data analysis and interpretation: JTG, MW, DH, PM and GB. Statistical analysis: JTG. Manuscript preparation: JTG. Manuscript editing: MW, DH and PM. Manuscript review: JTG, MW, DH and PM.

Funding This study was funded by an unrestricted grant from the Dutch Cancer Society (DCS).

Disclaimer The Dutch Cancer Society did not influence the article in any way. Competing interests None declared.

Patient consent for publication Not required.

Provenance and peer review Not commissioned; externally peer reviewed.

Data sharing statement № additional data are available.

Open access This is an open access article distributed in accordance with the Creative Commons Attribution Non Commercial (CC BY-NC 4.0) license, which permits others to distribute, remix, adapt, build upon this work non-commercially, and license their derivative works on different terms, provided the original work is properly cited, appropriate credit is given, any changes made indicated, and the use is non-commercial. See: http://creativecommons.org/licenses/by-nc/4.0/.

\section{REFERENCES}

1. Ghaferi AA, Birkmeyer JD, Dimick JB. Variation in hospital mortality associated with inpatient surgery. N Engl J Med 2009;361:1368-75.

2. Henneman D, Snijders HS, Fiocco M, et al. Hospital variation in failure to rescue after colorectal cancer surgery: results of the Dutch Surgical Colorectal Audit. Ann Surg Oncol 2013;20:2117-23.

3. Ghaferi AA, Birkmeyer JD, Dimick JB. Complications, failure to rescue, and mortality with major inpatient surgery in medicare patients. Ann Surg 2009;250:1029-34.

4. Finks JF, Osborne NH, Birkmeyer JD. Trends in hospital volume and operative mortality for high-risk surgery. N Engl J Med 2011;364:2128-37.
5. Almoudaris AM, Clark S, Vincent $C$, et al. Establishing quality in colorectal surgery. Colorectal Dis 2011;13:961-73.

6. Henneman D, van Leersum NJ, Ten Berge M, et al. Failure-to-rescue after colorectal cancer surgery and the association with three structural hospital factors. Ann Surg Oncol 2013;20:3370-6.

7. Ghaferi AA, Osborne NH, Birkmeyer JD, et al. Hospital characteristics associated with failure to rescue from complications after pancreatectomy. J Am Coll Surg 2010;211:325-30.

8. van Gijn W, Gooiker GA, Wouters MW, et al. Volume and outcome in colorectal cancer surgery. Eur J Surg Oncol 2010;36(Suppl 1):S55-63.

9. Lang TA, Hodge M, Olson V, et al. Nurse-patient ratios: a systematic review on the effects of nurse staffing on patient, nurse employee, and hospital outcomes. J Nurs Adm 2004;34:326-37.

10. Van Leersum NJ, Snijders HS, Henneman D, et al. The Dutch surgical colorectal audit. Eur J Surg Oncol 2013;39:1063-70.

11. Ghaferi AA, Dimick JB. Importance of teamwork, communication and culture on failure-to-rescue in the elderly. Br J Surg 2016;103:e47-51.

12. Healy DA, Doyle D, Moynagh E, et al. Systematic review and metaanalysis on the influence of surgeon specialization on outcomes following appendicectomy in children. Medicine 2015;94:e1352.

13. Taenzer AH, Pyke JB, McGrath SP. A review of current and emerging approaches to address failure-to-rescue. Anesthesiology 2011;115:421-31.

14. Rowlands C, Griffiths SN, Blencowe NS, et al. Surgical ward rounds in England: a trainee-led multi-centre study of current practice. Patient Saf Surg 2014;8:11.

15. Henneman D, Snijders HS, Fiocco M, et al. Hospital variation in failure to rescue after colorectal cancer surgery: results of the Dutch Surgical colorectal audit. Ann Surg Oncol 2013;20:2117-23.

16. Henneman D, Ten Berge MG, Snijders HS, et al. Safety of elective colorectal cancer surgery: non-surgical complications and colectomies are targets for quality improvement. J Surg Oncol 2014;109:567-73.

17. Kolfschoten NE, Marang-van de Mheen PJ, Wouters MW, et al. A combined measure of procedural volume and outcome to assess hospital quality of colorectal cancer surgery, a secondary analysis of clinical audit data. PLoS One 2014;9:e88737.

18. Silber JH, Rosenbaum PR, Schwartz JS, et al. Evaluation of the complication rate as a measure of quality of care in coronary artery bypass graft surgery. JAMA 1995;274:317.

19. SSI Web Sawtooth software. Version 7, 2016.

20. Archampong D, Borowski D, Wille-Jørgensen P, et al. Workload and surgeon's specialty for outcome after colorectal cancer surgery. Cochrane Database Syst Rev 2012:CD005391.

21. Huo YR, Phan K, Morris DL, et al. Systematic review and a metaanalysis of hospital and surgeon volume/outcome relationships in colorectal cancer surgery. J Gastrointest Oncol 2017;8:534-46.

22. Chioreso C, Del Vecchio N, Schweizer ML, et al. Association between hospital and surgeon volume and rectal cancer surgery outcomes in patients with rectal cancer treated since 2000: systematic literature review and meta-analysis. Dis Colon Rectum 2018;61:1320-32.

23. Jonker FHW, Hagemans JAW, Burger JWA, et al. The influence of hospital volume on long-term oncological outcome after rectal cancer surgery. Int J Colorectal Dis 2017;32:1741-7.

24. Jonker FHW, Hagemans JAW, Verhoef C, et al. The impact of hospital volume on perioperative outcomes of rectal cancer. Eur $J$ Surg Oncol 2017;43:1894-900.

25. Elferink MA, Wouters MW, Krijnen P, et al. Disparities in quality of care for colon cancer between hospitals in the Netherlands. Eur $J$ Surg Oncol 2010;36(Suppl 1):S64-73.

26. van Groningen JT, Eddes EH, Fabry HFJ, et al. Hospital Teaching Status and Patients' Outcomes After Colon Cancer Surgery. World J Surg 2018;42:3372-80.

27. Hall GM, Shanmugan S, Bleier JI, et al. Colorectal specialization and survival in colorectal cancer. Colorectal Dis 2016;18:051-60.

28. Oliphant R, Nicholson GA, Horgan PG, et al. Contribution of surgical specialization to improved colorectal cancer survival. Br J Surg 2013;100:1388-95.

29. Johnston MJ, Arora S, King D, et al. A systematic review to identify the factors that affect failure to rescue and escalation of care in surgery. Surgery 2015;157:752-63.

30. Johnston M, Arora S, King D, et al. Escalation of care and failure to rescue: a multicenter, multiprofessional qualitative study. Surgery 2014;155:989-94.

31. den Dulk M, Witvliet MJ, Kortram K, et al. The DULK (Dutch leakage) and modified DULK score compared: actively seek the leak. Colorectal Dis 2013;15:n/a-33.

32. den Dulk M, Noter SL, Hendriks ER, et al. Improved diagnosis and treatment of anastomotic leakage after colorectal surgery. Eur J Surg Oncol 2009;35:420-6. 
33. Arriaga AF, Lancaster RT, Berry WR, et al. The better colectomy project: association of evidence-based best-practice adherence rates to outcomes in colorectal surgery. Ann Surg 2009;250:507-13.

34. Keenan JE, Speicher PJ, Nussbaum DP, et al. Improving outcomes in colorectal surgery by sequential implementation of multiple standardized care programs. J Am Coll Surg 2015;221:404-14.
35. Wiebe ER, Kaczorowski J, MacKay J. Why are response rates in clinician surveys declining? Can Fam Physician 2012;58:e225-8.

36. Dobrow MJ, Orchard MC, Golden B, et al. Response audit of an Internet survey of health care providers and administrators: implications for determination of response rates. J Med Internet Res 2008;10:e30. 